\title{
Formation of double shell during implosion of plasma metal puff Z-pinches
}

\author{
Cite as: Phys. Plasmas 27, 092708 (2020); doi: 10.1063/5.0010853 \\ Submitted: 15 April 2020 . Accepted: 28 August 2020 . \\ Published Online: 22 September 2020
}

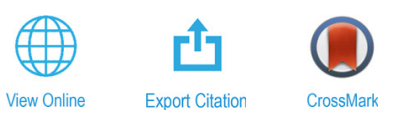

D. L. Shmelev, ${ }^{1,2, a)}$ (D) A. S. Zhigalin, ${ }^{3}$ (D) S. A. Chaikovsky, (D) V. I. Oreshkin, ${ }^{3,4}$ and A. G. Rousskikh ${ }^{3}$

\author{
AFFILIATIONS \\ ${ }^{1}$ Institute of Electrophysics, UB, RAS, 620016 Ekaterinburg, Russia \\ ${ }^{2}$ Ural Federal University, 620002 Ekaterinburg, Russian \\ ${ }^{3}$ Institute of High Current Electronics, SB, RAS, 634055 Tomsk, Russia \\ ${ }^{4}$ Tomsk Polytechnic University, 634050 Tomsk, Russia \\ a) Author to whom correspondence should be addressed: shmelev@iep.uran.ru
}

\begin{abstract}
This work presents the results of experimental and theoretical research of impact of tailored density profile and application of external axial magnetic field on initial spatial distribution of the plasma density in the plasma metal puff Z-pinch and on its implosion dynamics. It has been discovered that upon implosion of the plasma metal puff Z-pinch some stripes interpreted as the system of two coaxial shells appear on the optical images. With the help of numerical simulation, the formation of the plasma liner consisting of a mixture of carbon and bismuth ions and formed by the expansion of the plasma jet of the arc burning on the bismuth electrode has been considered in this work. It has been shown that the lightweight carbon ions facilitate formation of the density distribution smoothly decreasing with the increase in radius, that, in turn, leads to suppression of the Rayleigh-Taylor instability in the current sheath upon further implosion. It has also been demonstrated that availability of the two types of ions in plasma considerably different in mass leads to formation (in the compression phase) of a double shell with externally located heavy ions. It has also been revealed that the application of the external axial magnetic field leads to reduction in the plasma metal puff Z-pinch initial diameter.
\end{abstract}

Published under license by AIP Publishing. https://doi.org/10.1063/5.0010853

\section{INTRODUCTION}

The fast Z-pinches are quite widely researched presently both in terms of their application as a powerful source of soft $\mathrm{x}$-ray radiation and with regard to their possible use for inertial nuclear fusion. ${ }^{1-4}$ The interest to this problem in recent years is mainly connected with the magnetized liner inertial fusion program ${ }^{4,5}$ carried out in Sandia National Laboratories. A renewed interest is also present in the research of efficient multi-keV $\mathrm{x}$-ray generation, where large initial diameter $(>3 \mathrm{~cm})$ implosions are required. ${ }^{6-8}$

The compression of cylindrical fast Z-pinches takes place under the effect of a powerful current pulse creating high magnetic pressure on the pinches external surface. In the presence of the magnetic fields, the plasma compression is subject to magnetohydrodynamic instabilities, the most dangerous of which are Rayleigh-Taylor (RT) instabilities, which is why the fast Z-pinches (plasma liners) implosion stabilization is one of the most important aspects of their physics. ${ }^{1,9-15}$ To improve implosion stability, different approaches were proposed and implemented, particularly, use of axial magnetic field and snowplow stabilization mechanism. An effective way to suppress the RT instabilities in Z-pinches is the use of initial radial distribution of the substance density (tailored density profile ${ }^{9}$ ) at which the boundary between the magnetic field and plasma is not exposed to acceleration and the exponential growth of RT instability is fully suppressed. To suppress the RT instabilities, the authors of the work ${ }^{9}$ proposed to use such an initial distribution of the substance in the shell at which the density decreases as per the law $\rho(R) \sim R^{-s}$, where $\mathrm{s}>2$. Upon compression of the substance with such density distribution, the plasma boundary velocity begins to fall down after a short period of initial growth. One more consequence of the use of this kind of a profile is emergence of the low-density plasma on the periphery of the pinch. Ions with different charge-to-mass ratios can be separated in such plasma. This can be seen in the plasma consisting of a mixture of substances. ${ }^{14,15}$ In this case, the forces generated by the presence of gradients of magnetic field and thermal pressure in plasma have different impacts on the ions of different substances. These impacts are most pronounced in the presence of strong thermal pressure gradients and magnetic pressure gradients, i.e., in the current sheath and on the shock wave frontiers. ${ }^{16}$ 
Tailored density profiles have been implemented in experiments on plasma metal puff Z-pinch implosions. ${ }^{17-19}$ In one experiment, ${ }^{17}$ an axial magnetic field was not used and, in other experiments, ${ }^{18,19}$ implosion stabilization was achieved both with the help of the tailored density profile and with application of the external axial magnetic field. The metal puff Z-pinches are formed by injecting high-current vacuum arc plasma into a vacuum gap, the electrodes of which are under the voltage supplied by a generator with the current amplitude about a megampere to produce the Z-pinch. It was shown ${ }^{18}$ that the energy radiated from the $Z$-pinch can be considerably changed with the help of external axial magnetic field, the induction of which is much less than the induction of the Z-pinch self-magnetic field. It was demonstrated that the radiation energy of the bismuth Z-pinch compressed by IMRI- 5 generator $(450 \mathrm{kA}, 450 \mathrm{~ns})$ at $\mathrm{B}_{\mathrm{z} 0}=4.5 \mathrm{kGs}$ is about one and a half times as powerful as the Z-pinch energy without external magnetic field. The dynamics of Z-pinch compression and the total radiation output are mainly determined by initial spatial distribution of plasma liner density in the interelectrode gap. The aim of this article is complex experimental and theoretical research of impact of tailored density profile and application of external axial magnetic field both on initial spatial distribution of the plasma density in the plasma metal puff Z-pinch and on its implosion dynamics.

\section{EXPERIMENTAL SETUP AND PROCEDURE}

The experiments have been carried out on the high-current IMRI-5 pulse generator ${ }^{17-19}$ with the current amplitude (through the load) of about $450 \mathrm{kA}$ and a rise time of around $500 \mathrm{~ns}$. The scheme of experiments is presented in Fig. 1. The plasma produced by vacuumarc discharge was used as the load of the generator. The electrodes of the plasma gun (both cathode and anode) were made out of bismuth. The plasma jet was injected through a hole in the steel collimator, $5 \mathrm{~mm}$ in diameter. The length of the liner was $1 \mathrm{~cm}$ (the gap between the lowest electrode and the stainless-steel grid of IMRI-5 generator). It should be mentioned that the plasma injected from the plasma gun contained not only bismuth ions, of which the electrodes were made, but also the ions of isolator substance (mainly carbon ions ${ }^{20}$ ). All the experiments on the metal puff Z-pinch compression were carried out under the following fixed conditions: the delay between the beginning of the current flow in the arc discharge and switching the IMRI-5 generator current [ $450 \mathrm{kA}, 450 \mathrm{~ns}$ (Ref. 18)] was $\Delta t_{\mathrm{pl}}=6.2 \mu \mathrm{s}$; the voltage of the capacitor charging $\left(C_{\mathrm{pl}}=20 \mu \mathrm{F}\right)$ of the plasma source was

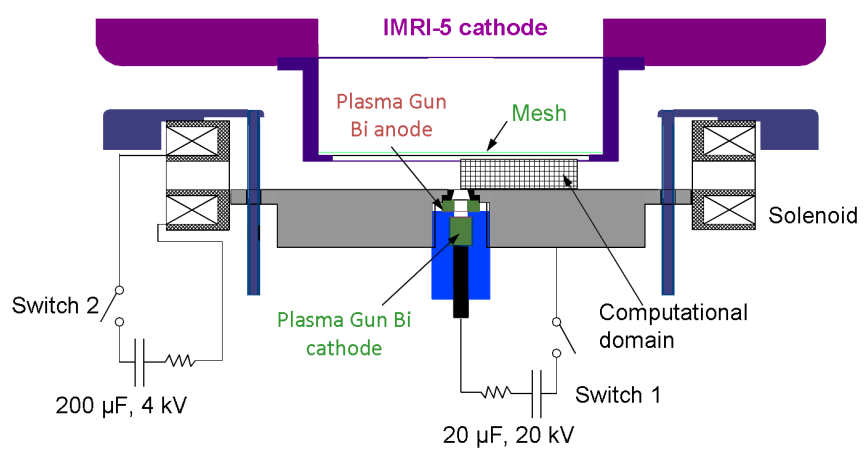

FIG. 1. Geometry of the problem.
$U_{\mathrm{pl}}=20 \mathrm{kV}$; a quarter of the current oscillation period in the arc discharge was $t_{1 / 4}=6.67 \mu \mathrm{s}$; and arc discharge current was $92 \pm 2 \mathrm{kA}$. Fixation of these conditions ensured a similar initial linear mass of the liners. It should, however, be mentioned that the application of the external magnetic field (see below) affected the plasma gun functioning, that, in turn, influenced the initial substance distribution in the interelectrode gap of IMRI-5 generator.

The external magnetic field was produced with the help of the two coils connected in series (Fig. 1). Each coil had 60 spirals. The capacitance in the magnetic field generation system was $\mathrm{C}_{\mathrm{bf}}=200 \mu \mathrm{F}$. The charge voltage of the system was $U_{\mathrm{bf}}=4 \mathrm{kV}$. The value of the magnetic field at the moment of switching on of the plasma source (and then IMRI-5 generator) was varied by changing the time interval $\Delta t_{\mathrm{bf}}$ between the beginning of the current flow in the magnetic field coil and the time of switching the rest of the system. The experiments were carried out at the following parameters of the magnetic field: $B_{1}$ $=1.5 \mathrm{kGs}\left(\Delta \mathrm{t}_{\mathrm{bf}}=47 \mu \mathrm{s}\right) ; B_{2}=3 \mathrm{kGs}\left(\Delta \mathrm{t}_{\mathrm{bf}}=114 \mu \mathrm{s}\right) ; B_{3}=4.5 \mathrm{kGs}$ $\left(\Delta t_{\mathrm{bf}}=200 \mu \mathrm{s}\right)$; and $\mathrm{B}_{4}=6 \mathrm{kGs}\left(\Delta \mathrm{t}_{\mathrm{bf}}=360 \mu \mathrm{s}\right)$.

It should be noted that the stabilization criteria for the pinch in the axial magnetic field can be presented as follows: ${ }^{8,21}$

$$
B_{z 0}(\kappa \mathrm{Gs}) \geq(10-30) \frac{I_{\max }(\mathrm{MA})}{R_{0}(\mathrm{~cm})},
$$

where $B_{z 0}$ is the initial axial magnetic field; $I_{\max }$ is the max current through the pinch; and $R_{0}$ is the initial radius. Criteria (1) reflects the fact that to improve the implosion stability at about tenfold compression the force accelerating the pinch and generated by the load current flowing through it should be balanced by the axial magnetic field pressure force. In the case of tailored density profile, it is quite difficult to identify the initial pinch radius. This question was discussed in Refs. 18 and 19, where the initial pinch radius was estimated as $\sim 3 \mathrm{~cm}$. Following this, the value $R_{0} \approx 3 \mathrm{~cm}$ can be accepted for crude evaluation. Then, according to Eq. (1), the magnetic field value required for the compression stabilization would be as follows: $B_{z 0} \geq(1.5-5) \mathrm{kGs}$. Thus, the external magnetic fields corresponding to (1) were used in the experiments.

The diagnostics during the experiments included both current and voltage measurements and registration of the images of the liners in their self-radiation. To obtain the plasma images in the visible spectral range, the HSFC Pro four-frame optical camera with the exposure of $3 \mathrm{~ns}$ was used. Current sheath boundary position upon plasma pinch compression was determined in the course of the experiments and detailed description of the measurement techniques is given in the paper. ${ }^{19}$

In the series of the experiments described in Ref. 19, the current sheath boundary position upon the plasma liner compression was determined using three different methods: by magnetic probe, by optical observation, and by measurement of the inductance $L(t)$ according to the following formula:

$$
R(t)=R_{r} \exp \left[-\frac{L(t)-L_{0}}{2}\right],
$$

where $R(t)$ is the sheath radius, $R_{r}$ is the reverse current posts radius, and $L_{0}$ is the inductance of the circuit section between the voltage gauge position and the Z-pinch. All three measurement methods showed comparable results. 
However, after performing the series of shots, it was discovered that the first shots in the series differed considerably from the consequent ones. The electrodes' initial contamination being eliminated after the first shot must have had a significant impact. In the case of regular "clean" shots, there were considerably less plasma in the liner, and the current shell initial radius was also lower; hence, it was difficult to determine the current sheath position with the use of magnetic probes because only one probe-the inner one-was operating in the standard manner in this case. Therefore, according to Ref. 19, the current sheath radius was derived by the inductance measurement in accordance with formula (2). Since the electrodes' contamination rate affects the Z-pinch current layer formation, four consequent shots were performed in each mode, wherein the plasma compression time changed due to reduced impurities and the image recording time was changed accordingly.

The pictures of the self-emission of the Z-pinches taken with the help of HSFC Pro optical camera revealed that there were the stripes parallel to the direction of the current flowing through the pinch (Fig. 2) on some images. The stripes were logical to associate with a system of two coaxial shells. These stripes or shells were the most clearly displayed ones when the pinch radius was approaching the value of about $1 \mathrm{~cm}$, and the distance between the stripes was around $1 \mathrm{~mm}$. This type of structure could be seen both in the absence and in the presence of the external magnetic field (Fig. 2). The nature and mechanism of emergence of the above structure we attempted to figure out in the course of numerical simulation, the methodology and results of which are described below.

\section{MODEL DESCRIPTION}

For simulation of the vacuum arc plasma jet expansion and the subsequent Z-pinch implosion, the two-dimensional hybrid model of quasi-neutral plasma was developed and tested. ${ }^{22-24}$ The model takes into account all three components of ion velocity, electron velocity, current, and magnetic field. However, all the components depend only on the two dimensions $r$ and $z$. This modification of the model allows studying the impact of the external axial magnetic field on the metal puff Z-pinch dynamics. The applicability of this code for the Z-pinch compression related problems was tested in. ${ }^{25,26}$ Here we will give only brief description of the main equation system.

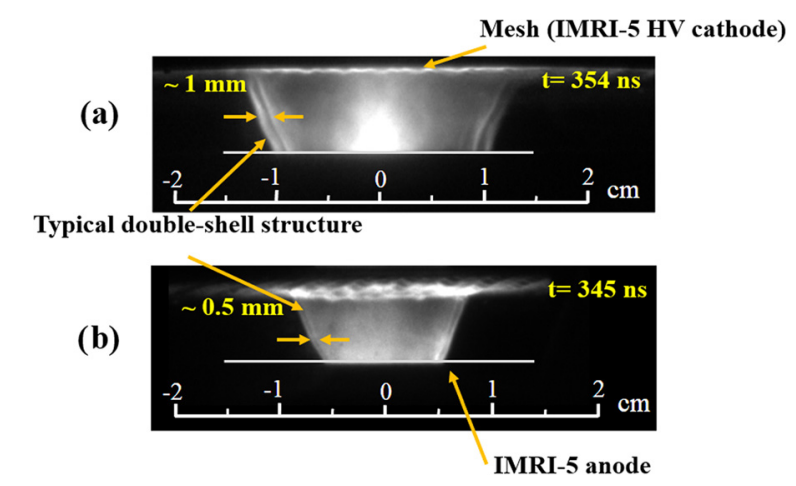

FIG. 2. Two parallel glowing shells (stripes) observed during the experiment. (a) Shot at $B_{z 0}=0 ; \mathrm{t}=354 \mathrm{~ns}$; estimated stripes thickness $\sim 1 \mathrm{~mm}$. (b) Shot at $B_{z 0}$ $=3$. $\mathrm{kGs} ; \mathrm{t}=345 \mathrm{~ns}$; estimated stripes thickness $\sim 0.5 \mathrm{~mm}$.
The model is a hybrid one, i.e., the ions are treated as the macroparticles with the help of the particles-in-cells (PICs) method [Eqs. (3)-(7)]. The macroparticles can describe the particles of different type, i.e., the particles having different charge and mass. The electrons are treated as the massless fluid (8), (9). The electromagnetic part in the quasi-neutral approximation is reduced to the equation for the transfer and diffusion of the magnetic field (only $\theta$-component) (10), transfer and diffusion of vector potential $\theta$-component (11), and the equation for the current and the magnetic field (12),

$$
\begin{aligned}
& \frac{d \vec{r}_{i}}{d t}=\vec{V}_{i}, \\
& m_{i} \frac{d \vec{V}_{i}}{d t}=e_{i} E+\frac{e_{i}}{c} \vec{V}_{i} \times \vec{B}+\left(\frac{\delta m_{i} \vec{V}_{i}}{\delta t}\right)_{i i}+\left(\frac{\delta m_{i} \vec{V}_{i}}{\delta t}\right)_{i e}, \\
& \vec{E}=-\frac{\vec{\nabla} P_{e}}{e n_{e}}-\frac{1}{c} \vec{u}_{e} \times \vec{B}-\frac{1}{n_{e}}\left\langle\frac{\delta m_{e} \vec{V}_{e}}{\delta t}\right\rangle, \\
& n_{i}=\frac{1}{H_{c}} \sum_{k=1}^{N_{c}} S\left(\Delta x_{k}\right), \quad \vec{u}_{i}=\frac{1}{H_{c}} \sum_{k=1}^{N_{c}} \vec{V}_{k} S\left(\Delta x_{k}\right), \\
& T_{i}=\frac{1}{H_{c}} \sum_{k=1}^{N_{c}} \frac{m_{i}}{3}\left(\vec{V}_{k}-\vec{u}_{i}\right)^{2} S\left(\Delta x_{k}\right), \\
& n_{\mathrm{e}}=\frac{1}{e} \sum_{i} e_{i} n_{i}, \quad \vec{u}_{e}=\frac{1}{e n_{e}}\left(\sum_{i} e_{i} n_{i} \vec{u}_{i}-\vec{J}\right), \\
& \frac{3}{2} n_{e}\left(\frac{\partial T_{e}}{\partial t}+\vec{u}_{e} \nabla T_{e}\right)+P_{e} \vec{\nabla} \cdot \vec{u}_{e}+\vec{\nabla} \chi \vec{\nabla} T_{e} \\
& =\left\langle\frac{1}{2} \frac{\delta m_{e}\left(\vec{V}_{e}-\vec{u}_{e}\right)^{2}}{\delta t}\right\rangle-W_{\text {ion }}-W_{\text {rad }} \\
& \frac{\partial B_{\theta}}{\partial t}+\frac{\partial u_{e z} B_{\theta}}{\partial z}+\frac{\partial u_{e r} B_{\theta}}{\partial r}-\frac{\partial u_{e \theta} B_{z}}{\partial z}-\frac{\partial u_{e \theta} B_{r}}{\partial r} \\
& =\frac{c^{2}}{4 \pi}\left(\frac{\partial}{\partial r}\left(\frac{1}{r \sigma} \frac{\partial r B_{\theta}}{\partial r}\right)+\frac{\partial}{\partial z}\left(\frac{1}{\sigma} \frac{\partial B_{\theta}}{\partial z}\right)\right), \\
& \frac{\partial A}{\partial t}+u_{e z} \frac{\partial A}{\partial z}+\frac{u_{e r}}{r} \frac{\partial r A}{\partial r}=\frac{c^{2}}{4 \pi} \frac{1}{\sigma}\left(\frac{\partial}{\partial r}\left(\frac{1}{r} \frac{\partial r A}{\partial r}\right)+\frac{\partial}{\partial z}\left(\frac{\partial A}{\partial z}\right)\right), \\
& B_{z}=\frac{1}{r} \frac{\partial r A}{\partial z}, B_{r}=-\frac{\partial A}{\partial z}, \quad \vec{J}=\frac{c}{4 \pi} \nabla \times \vec{B},
\end{aligned}
$$

where $V_{\mathrm{i}}$ is the velocity of the ion macroparticle of i-type, $r_{\mathrm{i}}$ is the radius vector of i-type macroparticle, $S$ is the weighting function of PIC method (the bilinear interpolation is presently used), $H_{\mathrm{c}}$ is the volume of PIC cell, $N_{\mathrm{c}}$ is the number of particles in cell, $n_{\mathrm{i}}$ is the density if i-type ions, $n_{\mathrm{e}}$ is the electrons density, $u_{\mathrm{i}}$ is the drift velocity of $\mathrm{i}$-type ions, $u_{\mathrm{e}}$ is the electrons drift velocity, $T_{\mathrm{e}}$ and $T_{\mathrm{i}}$ are the temperature of the electrons and ions, $J$ is the current density, $\tau_{\mathrm{ei}}$ is the electron-ion collision time, $\sigma$ is the plasma conductivity, $B$ is the magnetic field, $E$ is the electric field (in the context of quasi-neutrality), $P_{\mathrm{e}}$ is the electron pressure, $W_{\text {rad }}$ is the radiation losses, $W_{\text {ion }}$ is the losses of energy for ionization, and $A-\theta$ is the vector potential component.

The last two terms in the right-hand side of Eq. (4) formally indicate the variation of the macroparticles impulse caused by Coulomb ion-ion and electron-ion collisions, respectively. The first term in the right-hand side ( 9 ) is for the variation of electrons energy caused by such collisions. In the developed model, these collisions were simulated with the use of Monte Carlo method (MC). ${ }^{27}$ For ion-ion 
collisions simulation, the ions couples were randomly chosen in the cell. For ion-electrons collisions, the so-called grid-based binary model ${ }^{28}$ was used. The temporary set of the electrons subject to shifted Maxwell distribution with the grid values $n_{\mathrm{e}}, u_{\mathrm{e}}$, and $T_{\mathrm{e}}$ had been randomly sampled. Then the collisions between the ion macroparticles and the generated at each time step electron test macroparticles were simulated. ${ }^{24}$

The losses of energy for ionization $W_{\text {ion }}$ in Eq. (9) were calculated in the course of simulation of ionization and recombination reactions. The reactions were simulated with the help of direct simulation Monte Carlo method (DSMC). ${ }^{23,29}$ Direct ionization, triple recombination, and photo recombination were taken into account. The radiation losses $\mathrm{W}_{\text {rad }}$ in (9) were calculated in course of computation of radiation transfer with the use of P1 method. ${ }^{26,30}$

Compression dynamics was regulated by solving the following system of the electric circuit equations:

$$
\begin{gathered}
\left(L_{0}+L(t)\right) \frac{d I(t)}{d t}=-\left(R_{0}+R(t)+\frac{d L(t)}{d t}\right) I(t)-U(t), \\
\frac{d U(t)}{d t}=-\frac{I(t)}{C_{0}}, \\
L(t)=\frac{1}{I(t)^{2}} \int \frac{B(t)^{2}}{4 \pi} d V,
\end{gathered}
$$

where $\mathrm{L}_{0}$ is the initial circuit inductance, $\mathrm{C}_{0}$ is the capacitance, $\mathrm{U}$ is the voltage on the capacitor, $\mathrm{R}$ is the active liners resistance (was obtained in course of the computation), and I is the circuit current.

The simulation was made under the following circuit parameters: $\mathrm{L}_{0}=35 \mathrm{nH}, \mathrm{R}_{0}=60 \mathrm{~m} \Omega, \mathrm{C}_{0}=3.2 \mu \mathrm{F}$, and $\mathrm{U}(0)=70 \mathrm{kV}$. In the short circuit mode, the circuit parameters provided current $I(t)$ $=I_{\max } \cdot \sin \left(\left(\pi / 2 t_{0}\right) \cdot t\right)$, where $t_{0}$ was about $450 \mathrm{~ns}$, and $I_{\max }$ is the about $450 \mathrm{kA}$, which was in agreement with the current source characteristics used in the experiment.

The problem was solved in cylindrical geometry on a rectangular grid. The size of the computational domain is as follows: radius $=3.25 \mathrm{~cm}$, height $=1 \mathrm{~cm}$. The size of the computational cell of the grid varied upon the increase in the radius from $25 \mu \mathrm{m} \times 50 \mu \mathrm{m}$ to $90 \mu \mathrm{m} \times 50 \mu \mathrm{m}$. Because the computational domain was cylindrical, the macroparticles had different statistical weight that depended on radius of the particle injection into the computational domain. Macroparticles moving toward the center are split, thus increasing the number of the particles and reducing the chaotic fluctuations amplitude of the average values.

\section{COMPUTATION RESULTS}

In the course of the computation, we tried to obtain such a plasma distribution in the gap that would upon the liner compression make the current pulse reproduce the curve $\mathrm{R}(\mathrm{t})$ obtained during the experiment. $\mathrm{R}(\mathrm{t})$ was understood as a medium current sheath radius which, as in the experiment, was calculated according to formula (2), where $\mathrm{L}(\mathrm{t})$ was computed in the course of the liner compression simulation by Eq. (15). The fit to the experimental pinch compression curve was carried out in the absence of the external axial magnetic field. The boundary conditions for the plasma jet flowing from the vacuum-arc plasma source, obtained as a result of the above fitting, remained unchanged in the computation of the plasma expansion in the external magnetic field.
The expansion of the plasma jet entering the gap through the electrode center hole, $0.5 \mathrm{~cm}$ in diameter, was simulated at sinusoidal variation of arc current ( $80 \mathrm{kA}$, a quarter of the period) within $6.3 \mu \mathrm{s}$. At that, the specific erosion, temperature, and the composition of the plasma were varied for obtaining the required implosion curve. The vacuum-arc plasma gun with bismuth anode and dielectric insert were used in the experiments. The plasma composition of the source was quite complicated. ${ }^{18}$ In the present work, following the preliminary 0dimensional analysis with the use of the snow plow method, ${ }^{18}$ the arc plasma was assumed to be composed of bismuth and carbon. In the course of the simulation the following percentage composition was set: $\mathrm{Bi}-75 \%, \mathrm{C}-25 \%$; ion mean charge state $\mathrm{Bi}-1.5, \mathrm{C}-1$. Besides, it was assumed that $25 \%$ of the plasma ions were reflected (with neutralization) from the grid anode (grid transparency-50\%) and 50\% of the plasma ions were reflected from the cathode (at $\mathrm{R}>0.25 \mathrm{~cm}$ ).

The average velocity of the ions entering the gap was taken to be equal to $\mathrm{v}_{0}=5 \times 10^{5} \mathrm{~cm} / \mathrm{s}^{31}$ The plasma temperature in the course of the computer experiments was assumed to be $3 \mathrm{eV}$. At the above temperature, the bismuth ions thermal velocity was less then $\mathrm{v}_{0}$; hence, the ions showed weak expansion in the gap and formed the central dense jet. The carbon ions thermal velocity exceeded $\mathrm{v}_{0}$; therefore, the carbon ions expanded relatively fast forming light external shell.

In the course of the numerical simulation, it was revealed that the linear mass of the Z-pinch shall be $\sim 243 \mu \mathrm{g} / \mathrm{cm}$, most of it to be located close to the axis. To obtain this mass value under the given current, the specific erosion in the arc source shall be $\sim 1200 \mu \mathrm{g} / \mathrm{C}$ which is almost seven times as high as the experimental value obtained in the low-current arc on the bismuth cathode. ${ }^{32}$ However, there is some experimental data ${ }^{33}$ showing that the specific erosion in highcurrent arcs can repeatedly exceed the "conventional" values. Therefore, the obtained specific erosion value does not look to be extremely high. However, this problem (sharp growth of the specific erosion at high current) is worthy of a separate study, and this is the topic of one of our future works.

Figure 3 shows the comparison of the experimental pinch compression $R(\mathrm{t})$ curves developed by the inductance measurement according to formula (2) and the computed curves, as well as the oscillogram of the current flowing through the liner. Upon establishing the curve at $B_{z 0}=0$, the boundary conditions for the arc plasma jet were tuned. After that under the same boundary conditions, the computation was made at different $B_{z 0}$. It can be seen that, for example, there was quite a good correlation with the experimental curve at $B_{z 0}=3$ kGs.

The ions density distribution in the interelectrode gap at zero axial magnetic field is presented in Figs. 4 and 5. It can be seen that the density distribution in the gap has a profile smoothly decreasing with an increase in $R$. In the center of the gap at $R>0.25 \mathrm{~cm}$, the density behaves as follows: $n(R)\left(\mathrm{cm}^{-3}\right)=10^{18} \mathrm{e}^{-[3.5 R(\mathrm{~cm})]^{2}}$ $+3 \times 10^{15}[R(\mathrm{~cm})]^{-3.5}$, i.e., in the case of high radius values $R>0.7 \mathrm{~cm}$, the condition of RT instability suppression formulated in Ref. 9 is met. In Figs. 5 and 7, one can see that from the anode to the center of the gap such a profile (at high $\mathrm{R}$ values) is mainly formed by the carbon ions, and the bismuth ions create the central dense jet. It should be noted that a similar structure of the initial metal puff plasma Z-pinches substance distribution was observed in the experiments. ${ }^{20}$ The distribution of bismuth and carbon ions at the cathode is similar owing to a considerable number $(25 \%)$ of the ions reflected from the 


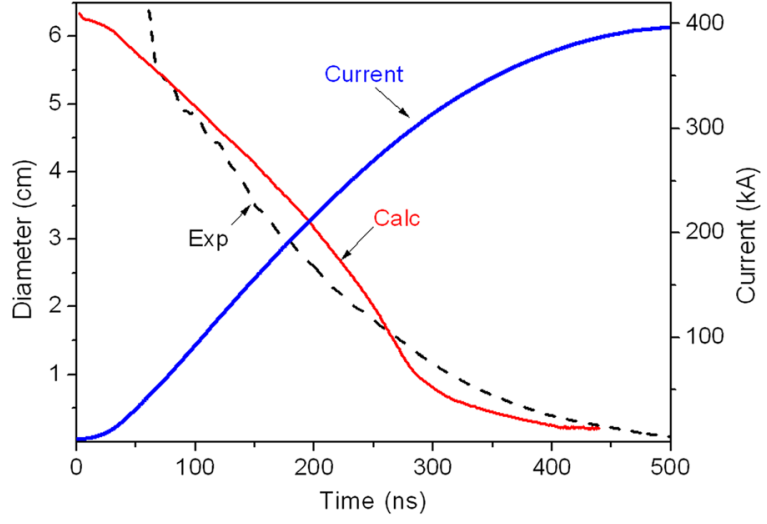

(a)

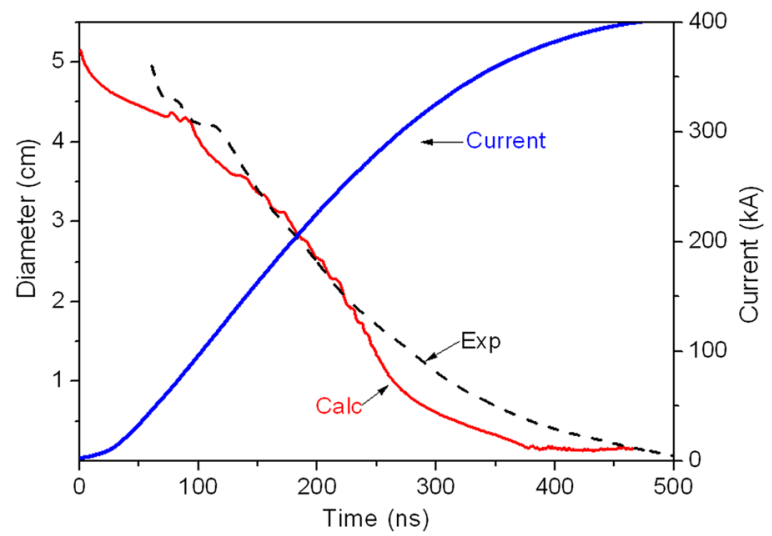

(b)

FIG. 3. Comparison of the plasma metal puff Z-pinch experimental compression $R(t)$ curve and the simulated $R(t)$ curve at different $B_{z 0}$. (a) $B_{z 0}=0 \mathrm{kGs}$ and (b) $B_{z 0}=3 \mathrm{kGs}$.

grid which mix with the ions approaching the anode. In spite of the fact that the carbon ions are injected in a three times lower quantity, they make the distribution of the aggregated density along $\mathrm{Z}$ at higher $R$ values more homogeneous. This positively influences the subsequent implosion dynamics.

According to Ref. 9, the Z-pinch density profile subject to power law of density reduction suppresses the development of Rayleigh-Taylor instability during the implosion of the Z-pinch that results in more homogeneous compression involving almost all

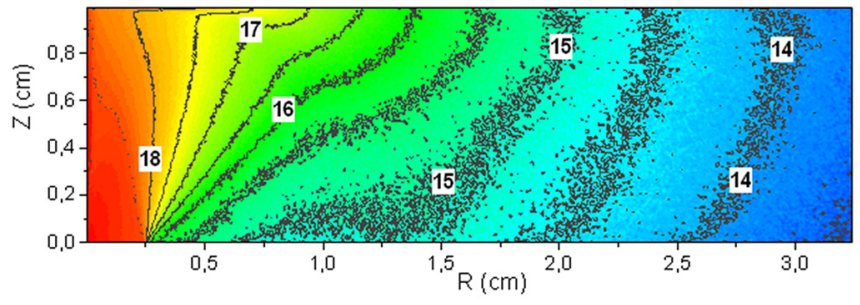

FIG. 4. Aggregated $(C+B i)$ ions density $\left[\lg (n), n\left(\mathrm{~cm}^{3}\right)\right] \cdot B_{z 0}=0$.

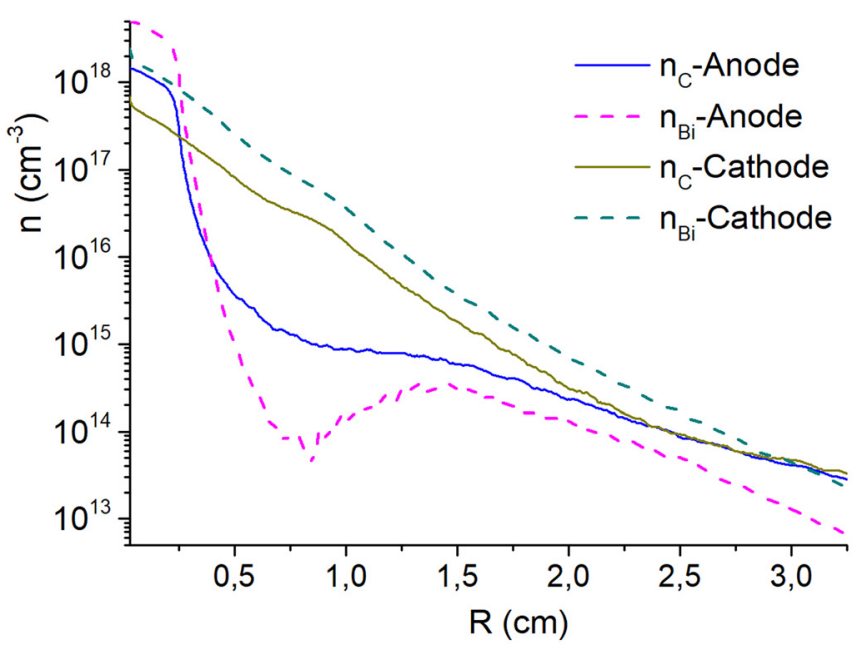

FIG. 5. Distribution of different component ions density in the gap. $B_{z 0}=0$.

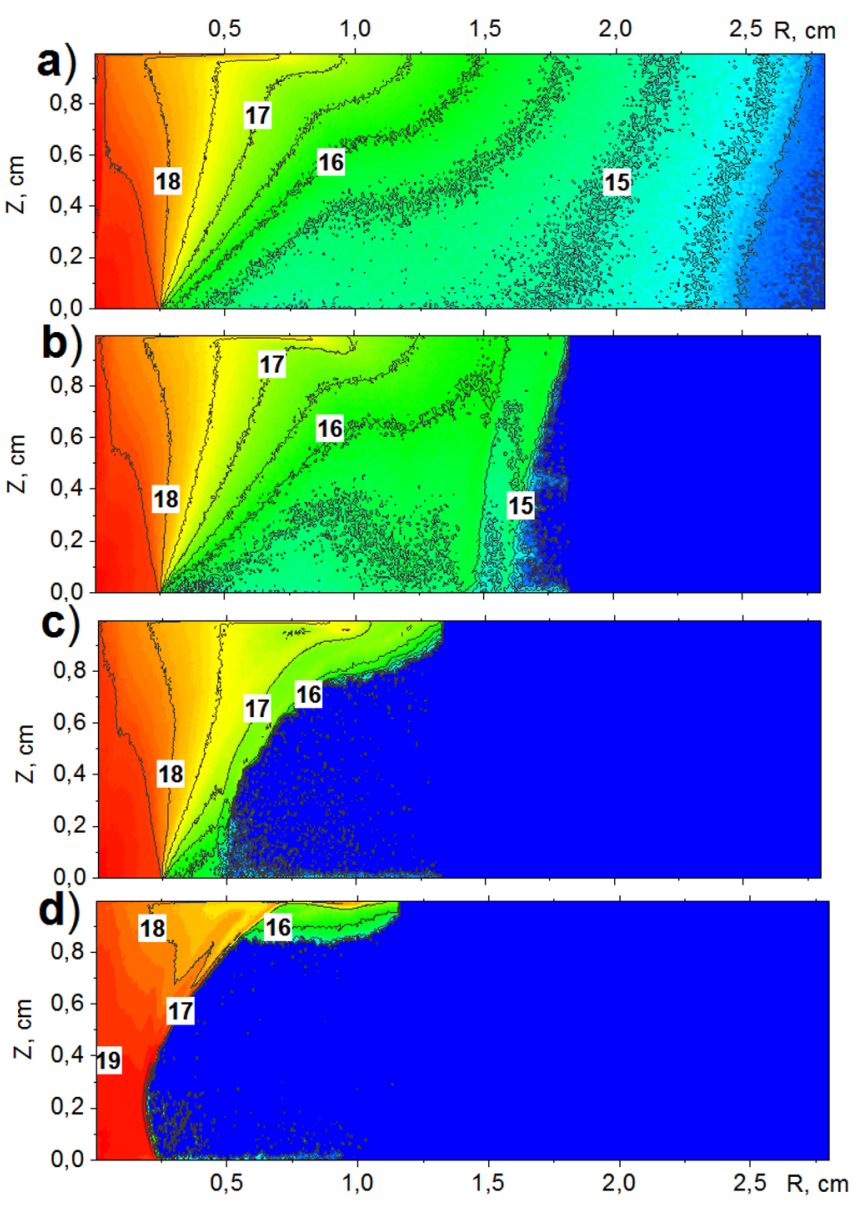

FIG. 6. Plasma liner compression. Aggregated $(C+B i)$ ions density $[\lg (n), n$ $\left(\mathrm{cm}^{3}\right)$ ] at different points of time. $B_{z 0}=1.5 \mathrm{kGs}$. (a) $\mathrm{t}=10 \mathrm{~ns}$, (b) $\mathrm{t}=200 \mathrm{~ns}$, (c) $\mathrm{t}=300 \mathrm{~ns}$, and (d) $\mathrm{t}=350 \mathrm{~ns}$. 
substance of the pinch. In the case of compression of a sharp boundary pinch, the Rayleigh-Taylor instabilities develop in the current sheath that makes only a part of the pinch substance to compress (see Refs. 11 and 34 for example).

As some experimental research ${ }^{18,19}$ reveal, the pinch formed by the above method is compressed homogeneously without any visible disturbances typical for the developed Rayleigh-Taylor instability. In our simulation the pinch also was compressed quite homogeneously without any instabilities in the current sheath (Fig. 6).

Figure 7 shows the density distribution in the center of the gap obtained at different values of the external axial magnetic field before the implosion begins. The plasma pinch initial radius decreases monotonously with the increase in $B_{z 0}$. It should be mentioned that during the computation it was assumed that the external magnetic field in the interelectrode gap was homogeneous at the beginning and had only Z-component. The actual axial magnetic field in the experimental installation is not homogeneous and has a nonzero $R$-component. But at this stage the task was simplified to discover main regularities of the external field influence.

In the course of the plasma expansion the initially homogeneous field deformed which resulted in the emergence of an azimuthal current and the resultant Ampère force decelerating the plasma expansion. Due to this, the plasma pinch initial radius decreased with the increase in $B_{z 0}$. So, at $B_{z 0}=6 \mathrm{kGs}$, the liner radius $\sim 1.8 \mathrm{~cm}$ against $3.25 \mathrm{~cm}$ at $B_{z 0}=0$. Figure 7 also reveals that at the tested $B_{z 0}$ values if $R<1 \mathrm{~cm}$ the plasma density distributions hardly vary.

In Fig. 7, one can see that at $B_{z 0}=0$ the Bi ion's density decreases with radius faster than the $\mathrm{C}$ ions density, and at $B_{z 0}>0$, there is the contrary situation. This is due to the fact that, as one can see from Eqs. (4)-(5), the forces driven by the presence of gradients of the thermal pressure and the magnetic field in plasma have different impact on different substances ions. The carbon ions being more lightweight are faster decelerated by the magnetic field, and the ion-ion interaction is weak due to the low plasma density and is not able to equalize the different mass ions velocities.

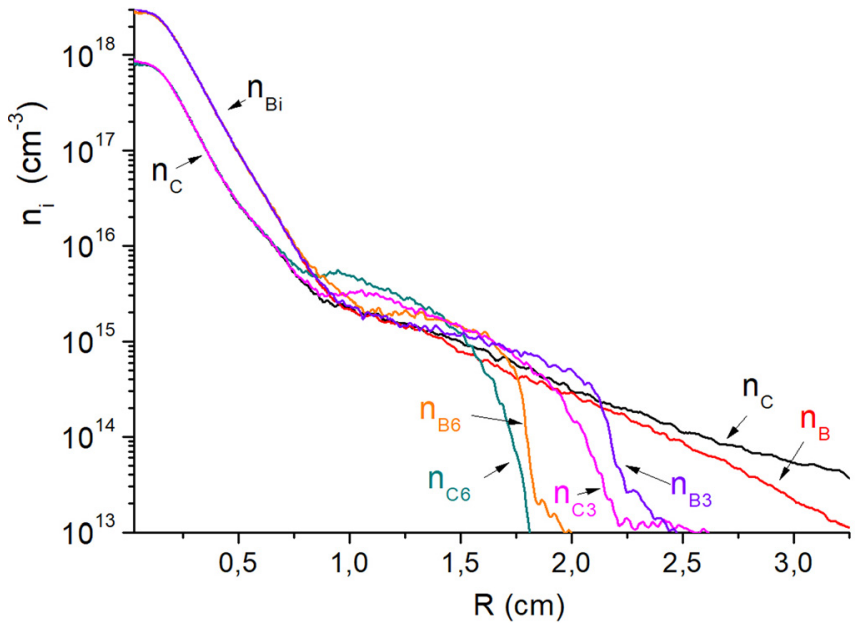

FIG. 7. Distribution of the ion densities at different external axial fields. " $C$ " and "B" stand for carbon and bismuth. The numbers 0,3 , and 6 mean that the computation was made at $B_{z 0}=0,3$, and $6 \mathrm{kGs}$, respectively.
The similar situation remains also upon implosion of the plasma pinch. But the carbon ions are accelerated more intensively than bismuth ions and the two parallel converging shells appear. Such shells can be seen in Figs. 6(b), 8, and 9, clearly demonstrating that such shells correspond to the local maxima of the different mass ions densities which are also accompanied by the local maxima of the current density and electron temperature. The distance between the density maxima in Fig. 9 can be related to the width of the double shell obtained in the experiment (Fig. 2). At $t=310$ ns, the width [Fig. 9(a)] is about $1 \mathrm{~mm}$, which is in good agreement with the experimental estimate [Fig. 2(a)]. The simulation has also revealed that the distance
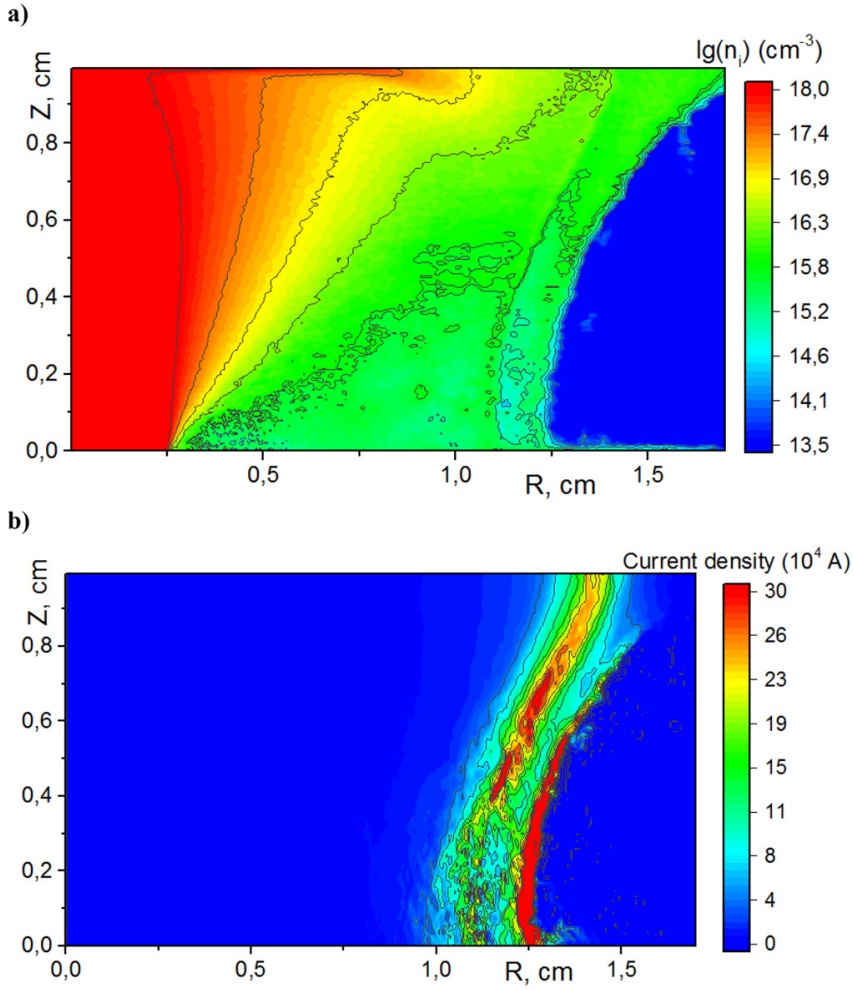

c)

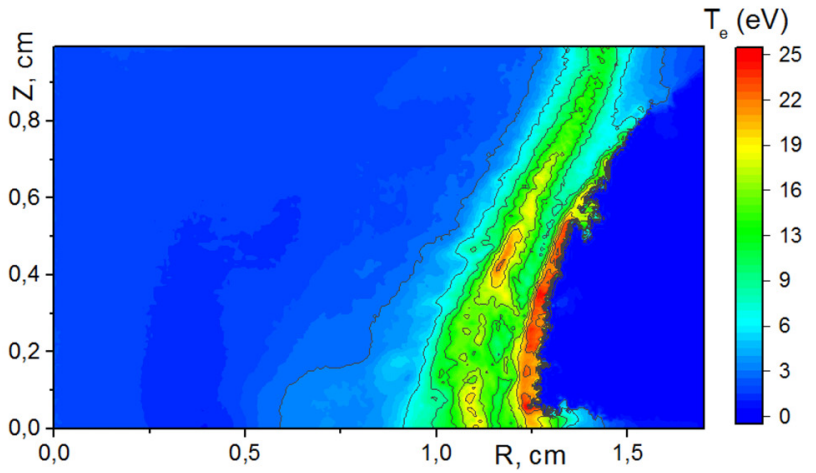

FIG. 8. (a) Aggregated density of plasma ions $\left[\mathrm{lg}(\mathrm{n}), \mathrm{n}\left(\mathrm{cm}^{3}\right)\right]$; (b) current density; and (c) electron temperature. $B_{z 0}=0, t=230 \mathrm{~ns}$. Two parallel shells can be seen at the external boundary. 


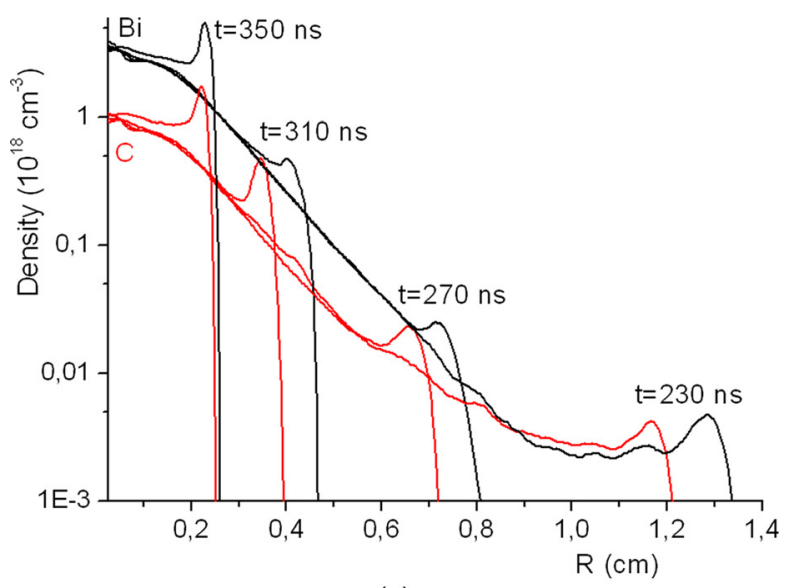

(a)

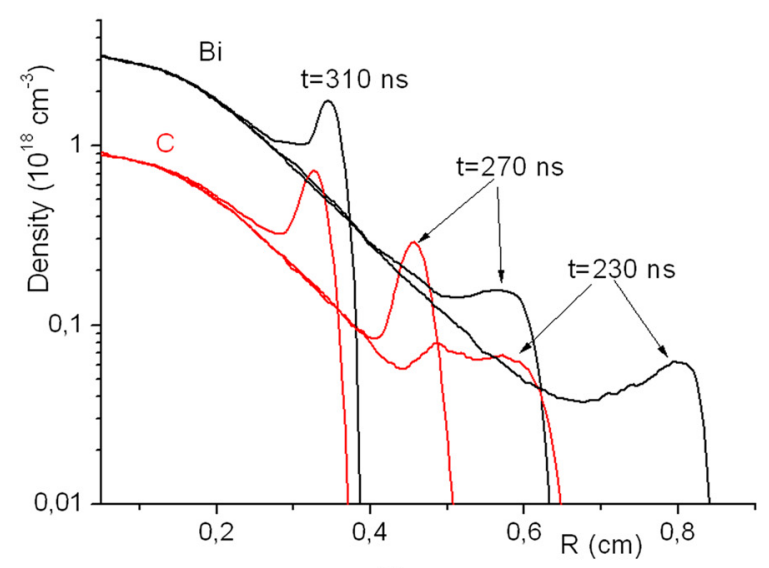

(b)

FIG. 9. Averaged ions densities distribution in the center of the gap at different points of time. (a) $B_{z 0}=0 \mathrm{kGs}$ and (b) $B_{z 0}=3 \mathrm{kGs}$.

between the shells changes in the course of the pinch compression (Fig. 9). When the compression starts the distance increases, the carbon ions move forward. When the shells approach the dense plasma area, the lightweight ions are decelerated more efficiently, and the distance between the shells decreases. Upon the further compression of the pinch, i.e., when the shells enters the dense plasma area, the efficiency of the ion-ion interaction enhances, the drift velocities of different mass ions equalize, and the two shells merge into one $[t=350 \mathrm{~ns}$, Fig. 9(a) and $t=310$ ns, Fig. 9(b)]. This kind of double luminous shells which merge into one at a later point of time during the compression is observed in the experiments.

It is necessary to mention that previously the formation of two concentric shells was observed ${ }^{14}$ and modeled $^{15}$ for multispecies $\mathrm{D}_{2}$-Ar gas puff. In Ref. 14 was shown that in the case of the $90 \%$ $\mathrm{D}_{2}-10 \%$ Ar mixture, a much better result for stabilization of implosion than in the case of pure $\mathrm{D}_{2}$ was achieved. However, the stability analysis conducted in Ref. 15 has indicated that radiative cooling from $\mathrm{Ar}$ ions has more to do with the improved pinches that are observed than with a reduction of the RT growth rate. In the case of present work, there is $\mathrm{Bi}$ ion liner with small admixture of $\mathrm{C}$ ions. The admixture of
$\mathrm{C}$ ions rather attenuates radiation cooling. Thus, the improvement in the implosion stabilization in our case is rather due to suppression of RT instability.

\section{CONCLUSION}

The work presents the results of experimental and theoretical research of impact of tailored density profile and external axial magnetic field on initial spatial distribution of the plasma metal puff Zpinch and on its implosion dynamics. The experiments have revealed that upon implosion of the metal puff Z-pinch some stripes parallel to the direction of the current flowing through the liner appear on the optical images obtained in their self-emission. The stripes have been interpreted as the system of two coaxial shells. Such structure is observed when the compressing pinch radius value approaches $1 \mathrm{~cm}$, and the "stripes" appear both in the absence and in the presence of the external magnetic field. The formation of the plasma pinch consisting of a mixture of carbon and bismuth ions as a result of the expansion of the arc plasma jet ignited on the bismuth electrode has been considered in the present study with the help of the numerical simulation. It has been revealed that the lightweight carbon ions facilitate generation of the tailored density profile which, in turn, provides suppression of the Rayleigh-Taylor instability in the current sheath upon the consequent implosion. It has also been demonstrated that availability of the two ion types considerably different in mass in the pinch plasma leads to formation of the double shells with externally located heavy ions in the compression phase. It has been shown that the application of the external axial magnetic field results in a decrease in initial diameter of the plasma metal puff $\mathrm{Z}$-pinch. The obtained results are in compliance with the experimental data.

\section{ACKNOWLEDGMENTS}

This work was funded by Russian Science Foundation. Project No. 19-19-00127.

\section{DATA AVAILABILITY}

The data that support the findings of this study are available from the corresponding author upon reasonable request.

\section{REFERENCES}

${ }^{\text {T} M . ~ G . ~ H a i n e s, ~ P l a s m a ~ P h y s . ~ C o n t r o l l e d ~ F u s i o n ~ 53, ~} 093001$ (2011). P.

2B. Jones, C. Deeney, C. A. Coverdale, P. D. LePell, J. L. McKeeney, J. P. Apruzese, J. W. Thornhill, K. G. Whitney, R. W. Clark, A. L. Velikovich, J. Davis, Y. Maron, V. Kantsyrev, A. Safonova, and V. I. Oreshkin, J. Quant. Spectrosc. Radiat. Transfer 99, 341-348 (2006).

${ }^{3}$ A. V. Branitskii, V. V. Aleksandrov, E. V. Grabovskii, V. V. Zazhivikhin, M. V. Zurin, S. F. Medovshchikov, S. L. Nedoseev, G. M. Oleinik, V. P. Smirnov, I. N. Frolov, and M. V. Fedulov, Plasma Phys. Rep. 25, 976-993 (1999).

${ }^{4}$ S. A. Slutz, M. C. Herrmann, R. A. Vesey, A. B. Sefkow, D. B. Sinars, D. C. Rovang, K. J. Peterson, and M. E. Cuneo, Phys. Plasmas 17, 056303 (2010).

${ }^{5}$ M. R. Gomez, S. A. Slutz, A. B. Sefkow, D. B. Sinars, K. D. Hahn, S. B. Hansen, E. C. Harding, P. F. Knapp, P. F. Schmit, C. A. Jennings et al., Phys. Rev. Lett. 113, 155003 (2014).

${ }^{6}$ C. A. Jennings, D. J. Ampleford, D. C. Lamppa, S. B. Hansen, B. Jones, A. J. Harvey-Thompson, M. Jobe, T. Strizic, J. Reneker, G. A. Rochau, and M. E. Cuneo, Phys. Plasmas 22, 056316 (2015).

${ }^{7}$ J. L. Giuliani and R. J. Commisso, IEEE Trans. Plasma Sci. 43, 2385-2453 (2015).

${ }^{8}$ H. Sze, J. Banister, B. H. Failor, J. S. Levine, N. Qi, A. L. Velikovich, J. Davis, D. Lojewski, and P. Sincerny, Phys. Rev. Lett. 95, 105001 (2005). 
${ }^{9}$ A. L. Velikovich, F. L. Cochran, and J. Davis, Phys. Rev. Lett. 77, 853-856 (1996).

${ }^{10}$ R. B. Bsksht, I. V. Datsko, and A. A. Kim, Plasma Phys. Rep. 21, 907-912 (1995).

${ }^{11}$ M. R. Douglas, J. S. De Groot, and R. B. Spielman, Laser Part. Beams 19, 527 (2001).

${ }^{12}$ A. Y. Labetsky, R. B. Baksht, V. I. Oreshkin, A. G. Rousskikh, and A. V. Shishlov, IEEE Trans. Plasma Sci. 30, 524-531 (2002).

${ }^{13}$ H. Sze, J. S. Levine, J. Banister, B. H. Failor, N. Qi, and P. Steen, Phys. Plasmas 14, 056307 (2007).

${ }^{14}$ J. Bailey, Y. Ettinger, A. Fisher, and N. Rostoker, Appl. Phys. Lett. 40, 460-462 (1982).

${ }^{15}$ H. U. Rahman, P. Amendt, and N. Rostoker, Phys. Fluids 28, 1528-1531 (1985).

${ }^{16}$ V. I. Oreshkin, "Diffusion in multicomponent liners," AIP Conf. Proc. 409, 215-218 (1997).

${ }^{17}$ A. G. Rousskikh, A. S. Zhigalin, V. I. Oreshkin, N. A. Labetskaya, S. A. Chaikovsky, A. V. Batrakov, G. Yu. Yushkov, and R. B. Baksht, Phys. Plasmas 21, 052701 (2014).

${ }^{18}$ A. G. Rousskikh, A. S. Zhigalin, V. I. Oreshkin, V. Frolova, A. L. Velikovich, G. Y. Yushkov, and R. B. Baksht, Phys. Plasmas 23, 063502 (2016).

${ }^{19}$ A. G. Rousskikh, A. S. Zhigalin, V. I. Oreshkin, and R. B. Baksht, Phys. Plasmas 24, 063519 (2017).

${ }^{20}$ A. G. Rousskikh, A. S. Zhigalin, and V. I. Oreshkin, Tech. Phys. 61, 676-682 (2016).
${ }^{21}$ A. B. Bud'ko, A. L. Velikovich, M. A. Liberman, and F. S. Felber, Zh. Eksp. Teor. Fiz. 96, 496 (1989) (in Russian).

${ }^{22}$ D. L. Shmelev and I. V. Uimanov, IEEE Trans. Plasma Sci. 43, 2261-2266 (2015).

${ }^{23}$ D. L. Shmelev, V. I. Oreshkin, and I. V. Uimanov, IEEE Trans. Plasma Sci. 47, 3478-3483 (2019).

${ }^{24}$ D. L. Shmelev, I. V. Uimanov, and L. Wang, 4th International Conference on Electric Power Equipment-Switching Technology (ICEPE-ST) (IEEE Xplore, 2017).

${ }^{25}$ D. L. Shmelev, V. I. Oreshkin, and S. A. Chaikovsky, J. Phys.: Conf. Ser. 830, 012018 (2017).

${ }^{26}$ D. L. Shmelev, V. I. Oreshkin, and S. A. Chaikovsky, J. Phys.: Conf. Ser. 1115, 022014 (2018).

${ }^{27}$ A. V. Bobylev and I. F. Potapenko, J. Comp. Phys. 246, 123-144 (2013).

${ }^{28}$ B. I. Cohen, A. M. Dimits, and D. J. Strozzi, J. Comp. Phys. 234, 33-43 (2013).

${ }^{29}$ V. V. Serikov, S. Kawamoto, and K. Nambu, IEEE Trans. Plasma Sci. 27, 1389-1398 (1999).

${ }^{30}$ M. F. Modest, Radiative Heat Transfer (McGraw-Hill, New York, 1993).

${ }^{31}$ G. Yu. Yushkov, A. Anders, E. M. Oks, and I. G. Brown, J. Appl. Phys. 88, 5618 (2000).

${ }^{32}$ A. Anders, E. M. Oks, G. Y. Yushkov, K. P. Savkin, I. G. Brown, and A. G. Nikolaev, IEEE Trans. Plasma Sci. 33, 1532-1536 (2005).

${ }^{33}$ A. G. Rousskikh, R. B. Baksht, A. S. Zhigalin, V. I. Oreshkin, S. A. Chaikovsky, and N. A. Labetskaya, Plasma Phys. Rep. 38(8), 595-607 (2012).

${ }^{34}$ D. R. Welch, D. V. Rose, C. Thoma, R. E. Clark, C. B. Mostrom, W. A. Stygar, and R. J. Leeper, Phys. Plasmas 17, 072702 (2010). 